\title{
Canonical formalism of nonrelativistic theories coupled to Newton-Cartan gravity
}

\author{
Josef Klusoň \\ Department of Theoretical Physics and Astrophysics, Faculty of Science, Masaryk University, \\ Kotlářská 2, 611 37, Brno, Czech Republic
}

(Received 14 July 2018; published 24 September 2018)

\begin{abstract}
In this paper we perform a canonical analysis of the Schrödinger field and nonrelativistic electrodynamics coupled to Newton-Cartan gravity. We identify physical degrees of freedom and analyze the constraint structure of these theories.
\end{abstract}

DOI: 10.1103/PhysRevD.98.066014

\section{INTRODUCTION AND SUMMARY}

There has been a renewed interest in Newton-Cartan (NC) geometry in recent years. The first significant paper was Ref. [1] which introduced NC geometry to field theory to analyze strongly correlated electrons. It was further shown in Refs. [2,3] that NC geometry with torsion naturally emerges as the background boundary geometry in holography for $z=2$ Lifshitz geometries; for relevant works, see Refs. [4-7] and for a review and extensive list of references, see Ref. [8]. In fact, NC geometry is a nonrelativistic background geometry to which nonrelativistic field theories can be covariantly coupled; see e.g., Refs. [5,9-13]. In particular, it was shown in a significant paper [11] how nonrelativistic electrodynamics can couple to the most general NC geometry with torsion. Further, nonrelativistic scalar fields coupled to NC geometry and a background electromagnetic field were also analyzed there.

Since these results are very interesting nonrelativistic theories in the NC background certainly deserve to be studied in more detail. In this paper we focus on a canonical analysis of nonrelativistic scalar fields and nonrelativistic electrodynamics coupled to $\mathrm{NC}$ geometry. ${ }^{1}$ It turns out that this is a rather nontrivial problem with the interesting property that the constraints explicitly depend on time. In more detail, we start with the action for the Schrödinger field in the background $\mathrm{NC}$ geometry and a background nonrelativistic electromagnetic field. Such an action was derived in Ref. [11] with the help of the null reduction of a

\footnotetext{
*klu@physics.muni.cz

${ }^{1}$ For a canonical analysis of Carrollian electrodynamics, see Ref. [14].

Published by the American Physical Society under the terms of the Creative Commons Attribution 4.0 International license. Further distribution of this work must maintain attribution to the author(s) and the published article's title, journal citation, and DOI. Funded by SCOAP ${ }^{3}$.
}

complex scalar field in higher-dimensional space-time. ${ }^{2}$ Clearly the canonical form of the action could be derived for a general NC space-time, at least in principle; however we restrict ourselves to a simpler situation which has a clear physical meaning. More precisely, we impose an important restriction on the NC space-time in the sense that it has to have a notion of foliation by spatial surfaces that are orthogonal to the one-form $\tau_{\mu}$ where $\tau_{\mu}$ is known as the clock form. This form defines a preferred notion of spatial direction at each point and also an arrow of time in the sense that the vector field $t^{\mu}$ is said to be future directed if it obeys the condition $\tau_{\mu} t^{\mu}>0 . \tau_{\mu}$ defines a pointwise notion of spatial direction with the help of the vectors $w^{\mu}$ that obey the condition $\tau_{\mu} w^{\mu}=0$. However this notion can be integrated to a local codimension-one subspace when $\tau_{\mu}$ obeys the Frobenius condition $\tau \wedge d \tau=0$ where $\tau=\tau_{\mu} d x^{\mu}$. Then we define causal space-times as spacetimes where this condition holds everywhere; for a more detailed analysis and discussion, see e.g., Refs. [9,12]. For such a space-time we will be able to find the Hamiltonian for the Schrödinger field in the NC background. However we also find that when we write the complex scalar field in polar form as $\psi=\sqrt{\rho} e^{i S}$ the momentum conjugate to $\rho$ is zero which is the first primary constraint of the theory. Further, we find that the momentum conjugate to $S$ is determined by the second primary constraint that explicitly depends on time. This is a very interesting situation that deserves to be treated carefully. For that reason we perform an analysis of constraint systems with explicit time-dependent primary constraints in the Appendix. ${ }^{3}$ Taking into account the explicit time dependence of the constraints we will be able to derive canonical equations of motion that

\footnotetext{
${ }^{2}$ Null reduction was studied in some earlier papers $[15,16]$.

${ }^{3} \mathrm{~A}$ discussion of the constraint analysis with explicit time dependence can be found in Ref. [17]; however the analysis presented there is slightly different from ours.
} 
reproduce the Lagrangian equations of motion which is a nice consistency check.

As the next step we extend this analysis to the case of nonrelativistic electrodynamics in the Newton-Cartan background. Since canonical analysis is based on an existence of Lagrangian we start with the nonrelativistic electrodynamics action in the $\mathrm{NC}$ background that is derived using null-dimensional reduction [11]. We again restrict ourselves to the case of causal space-time and in the first step we determine a set of primary constraints which Poisson commute among themselves. This is a different situation than in the case of the scalar field where the primary constraints were the second class constraints. Then the requirement of the preservation of the primary constraints gives a set of secondary constraints which together with the primary constraints form the set of second class constraints. As a result we find that the gauge field and corresponding conjugate momenta can be eliminated from the theory at least in principle. We also determine Lagrange multipliers corresponding to the primary constraints using the equations of motion for the gauge field and we show that the resulting equations of motion coincide with the equations of motion derived by varying the action.

Let us outline the main results derived in this paper. We obtain the Hamiltonian form of nonrelativistic theories on the NC background and we determine the physical degrees of freedom (d.o.f.). This is a very important result since we show that in the case of nonrelativistic electrodynamics the only physical d.o.f. are the scalar field and conjugate momenta. We also discuss the problem of the constraint structure in the case of theories with explicit time-dependent constraints.

The structure of this paper is as follows. In Sec. II we review basic facts about NC geometry and introduce an action for the Schrödinger field in the NC background and background nonrelativistic electromagnetic field through null-dimensional reduction. Then we perform a Hamiltonian analysis of this theory and determine the structure of the constraints. In Sec. III we analyze nonrelativistic electrodynamics in the NC background. We first perform a canonical analysis of nonrelativistic electrodynamics in a flat background and then we extend this analysis to the case of nonrelativistic electrodynamics in the NC background. Finally in the Appendix we study constrained systems with explicit time dependence and discuss their properties.

\section{HAMILTONIAN ANALYSIS OF THE SCHRÖDINGER FIELD IN THE NC BACKGROUND}

\section{A. Summary of Newton-Cartan geometry}

We start this analysis with a brief review of NewtonCartan geometry in $d+1$ dimensions. The Newton-Cartan background in $d+1$ dimensions is given by a set of one-forms $\left(\tau_{\mu}, e_{\mu}{ }^{a}\right)$ where $a=1, \ldots, d$ and where $\mu, \nu=0,1, \ldots, d$. We also have the one-form $M_{\mu}$. We define inverse vielbeins $v^{\mu}$ and $e_{\mu}{ }^{a}$ through the relations

$v^{\mu} e_{\mu}{ }^{a}=0, \quad v^{\mu} \tau_{\mu}=-1, \quad e^{\mu}{ }_{a} \tau_{\mu}=0, \quad e^{\mu}{ }_{a} e_{\mu}{ }^{b}=\delta_{a}^{b}$.

The determinant of the $(d+1) \times(d+1)$ matrix $\left(\tau_{\mu}, e_{\mu}{ }^{a}\right)$ is denoted by $e$. With the help of vierbeins we can construct a degenerative "spatial metric"

$$
h_{\mu \nu}=e_{\mu}{ }^{a} e_{\nu}{ }^{b} \delta_{a b}, \quad h^{\mu \nu}=e^{\mu}{ }_{a} e^{\nu}{ }_{b} \delta^{a b} .
$$

By definition, the one-forms $\tau_{\mu}, e_{\mu}{ }^{a}$ and $M_{\mu}$ transform under a diffeomorphism as usual but they also transform under various local transformations: Galilean boosts with $\lambda_{a}$ as the local parameter, local $S O(d)$ rotations which are parametrized by $\lambda_{a b}=-\lambda_{b a}$ and the $U(1)_{\sigma}$ gauge transformation which is parametrized by $\sigma$ where we have

$$
\begin{aligned}
\delta \tau_{\mu} & =0, \quad \delta e_{\mu}{ }^{a}=\tau_{\mu} \lambda^{a}+\lambda^{a}{ }_{b} e_{\mu}{ }^{b}, \\
\delta v^{\mu} & =\lambda^{a} e^{\mu}{ }_{a}, \quad \delta e^{\mu}{ }_{a}=\lambda_{a}{ }^{b} e^{\mu}{ }_{b}, \\
\delta M_{\mu} & =\lambda_{a} e_{\mu}{ }^{a}+\partial_{\mu} \sigma .
\end{aligned}
$$

The inverse vielbein $e^{\mu}{ }_{a}$ is invariant under local Galilean transformations. Note that we have an important relation

$$
e_{\mu}{ }^{a} e^{\nu}{ }_{a}-\tau_{\mu} v^{\nu}=\delta_{\mu}^{\nu}
$$

which implies

$$
h_{\mu \nu} h^{\nu \rho}=\delta_{\mu}^{\rho}+\tau_{\mu} v^{\rho}
$$

which will be useful below. It is also useful to define the objects $\hat{v}^{\mu}, \hat{e}_{a}^{\mu}, \hat{h}^{\mu \nu}$ and $\Phi$ which are invariant under local Galilean transformations:

$$
\begin{aligned}
& \hat{v}^{\mu}=v^{\mu}-h^{\mu \nu} M_{\nu}, \quad \hat{e}_{a}^{\mu}=e_{a}^{\mu}-M_{\nu} e_{b}^{\nu} \delta^{b a} \tau_{\mu}, \\
& \hat{h}_{\mu \nu}=h_{\mu \nu}-M_{\mu} \tau_{\nu}-M_{\nu} \tau_{\mu}, \quad \Phi=-v^{\mu} M_{\mu}+\frac{1}{2} h^{\mu \nu} M_{\mu} M_{\nu} .
\end{aligned}
$$

It is important to stress that $\hat{h}_{\mu \nu} \neq \hat{e}_{\mu}{ }^{a} \hat{e}_{\nu}{ }^{b} \delta_{a b}$. Instead, using the definition of $\hat{e}_{\mu}{ }^{a}$ given above, we obtain the following relation:

$$
\hat{e}_{\mu}{ }^{a} \hat{e}_{\nu}{ }^{b} \delta_{a b}=\hat{h}_{\mu \nu}+2 \tau_{\mu} \tau_{\nu} \Phi .
$$

Finally we note that hatted objects obey the following relations:

$$
\hat{v}^{\mu} \hat{e}_{\mu}{ }^{a}=0, \quad \hat{v}^{\mu} \tau_{\mu}=-1, \quad e^{\mu}{ }_{a} \hat{e}_{\mu}{ }^{b}=\delta_{a}^{b} .
$$


After this review of $\mathrm{NC}$ geometry we proceed to the Hamiltonian analysis of the Schrödinger field.

\section{B. Schrödinger field in NC geometry through null-imensional reduction}

We would like to find the Hamiltonian formulation of the scalar field on the Newton-Cartan background with a fixed electromagnetic background. The most convenient way to find such an action is to perform null-dimensional reduction; see e.g., Refs. $[9,11]$. Let us consider an action for a complex scalar field in $d+2$ dimensions in the form

$$
I=\int d^{d+2} x \sqrt{-\gamma}\left(-\gamma^{A B} D_{A} \Psi D_{B} \Psi^{*}\right),
$$

where $D_{A}=\partial_{A} \Psi-i q A_{A} \Psi$ and where $A_{A}, A=0, \ldots, d+1$ is the background electromagnetic field. Let us now consider a background metric that possesses a null isometry that is generated by coordinates $\partial_{u}$

$$
d s^{2}=\gamma_{A B} d x^{A} d x^{B}=2 \tau_{\mu} d x^{\mu}\left(d u-M_{\nu} d x^{\nu}\right)+h_{\mu \nu} d x^{\mu} d x^{\nu}
$$

so that

$$
\gamma_{\mu u}=\gamma_{u \mu}=\tau_{\mu}, \quad \gamma_{\mu \nu}=h_{\mu \nu}-\tau_{\mu} M_{\nu}-\tau_{\nu} M_{\nu} \equiv \hat{h}_{\mu \nu} .
$$

Then

$$
\sqrt{-\gamma}=e, \quad e=\operatorname{det}\left(\tau_{\mu}, e_{\mu}{ }^{a}\right) .
$$

Since the metric $\gamma_{A B}$ is nonsingular we can easily find the inverse metric with components

$$
\gamma^{u u}=2 \Phi, \quad \gamma^{u \mu}=-\hat{v}^{\mu}, \quad \gamma^{\mu \nu}=h^{\mu \nu},
$$

where $\hat{v}^{\mu}$ and $\Phi$ are defined in Eq. (6). We further presume that the gauge field has the form $A_{A}=\left(A_{u}, A_{\mu}\right)=$ $\left(\varphi, \bar{A}_{\mu}-\varphi M_{\mu}\right)$. Now with the help of this metric we perform a twisted null reduction when the action does not depend on $u$. Then it is clear that all metric components do not depend on $u$ while $\Psi$ can have the form

$$
\Psi=e^{i m u} \psi,
$$

where $\psi$ does not depend on $u$. Then by inserting Eq. (14) into the action and using Eq. (13) we obtain

$$
\begin{aligned}
I= & \int d^{d+1} x e\left(\hat{v}^{\mu}\left(D_{\mu} \psi\right)^{*} i(m-q \varphi) \psi-i(m-q \varphi) \hat{v}^{\mu} D_{\mu} \psi \psi^{*}\right. \\
& \left.-2 \Phi(m-q \varphi)^{2} \psi \psi^{*}-h^{\mu \nu} D_{\mu} \psi\left(D_{\nu} \psi\right)^{*}\right),
\end{aligned}
$$

where

$$
D_{\mu} \psi=\partial_{\mu} \psi-i q A_{\mu} \psi, \quad\left(D_{\mu} \psi\right)^{*}=\partial_{\mu} \psi^{*}+i q A_{\mu} \psi^{*} .
$$

The action (15) is the action for the Schrödinger field with mass $m$ and charge $q$ in the Newton-Cartan background and in the background electromagnetic field where the electromagnetic field has components $A_{\mu}$. Note that $\psi$ couples to $\varphi$ through the combination $m-q \varphi$ and hence $\varphi$ effectively shifts the mass of the scalar field and hence it is natural to call it the mass potential [11]. Our goal is to find the Hamiltonian from the action (15).

\section{Hamiltonian analysis}

We would like to work with real variables rather than with complex ones. For that reason we parametrize the scalar field $\psi$ as $\psi=\sqrt{\rho} e^{i S}$. In this case we find that the covariant derivative $D_{\mu} \psi$ has the form

$$
\begin{aligned}
D_{\mu} \psi & =\left[\frac{1}{2 \sqrt{\rho}} \partial_{\mu} \rho+i \sqrt{\rho}\left(\partial_{\mu} S-q A_{\mu}\right)\right] e^{i S}, \\
\left(D_{\mu} \psi\right)^{*} & =\left[\frac{1}{2 \sqrt{\rho}} \partial_{\mu} \rho-i \sqrt{\rho}\left(\partial_{\mu} S-q A_{\mu}\right)\right] e^{-i S}
\end{aligned}
$$

so that the action has the form

$$
\begin{aligned}
I^{\mathrm{sch}}= & \int d^{d+1} x e\left(2(m-q \varphi) \rho \hat{v}^{\mu}\left(\partial_{\mu} S-q A_{\mu}\right)\right. \\
& -2 \Phi(m-q \varphi) \rho-\frac{1}{4 \rho} h^{\mu \nu} \partial_{\mu} \rho \partial_{\nu} \rho \\
& \left.-h^{\mu \nu} \rho\left(\partial_{\mu} S-q A_{\mu}\right)\left(\partial_{\nu} S-q A_{\nu}\right)\right) .
\end{aligned}
$$

We see that this action is manifestly invariant under the gauge transformations

$S^{\prime}(x)=S(x)+q \Lambda(x), \quad A_{\mu}^{\prime}(x)=A_{\mu}(x)+\Lambda(x)$.

It is clear that the previous action is well defined for a general Newton-Cartan background for an arbitrary $\tau_{\mu}$ apart from the fact that $\tau_{\mu}$ has to obey the Newton-Cartan compatibility condition. On the other hand in order to have a well-defined Hamiltonian formulation we have to have a notion of foliation by spatial surfaces that are orthogonal to $\tau_{\mu}$. This is guaranteed when we impose the hypersurface orthogonality condition $\tau_{[\mu} \partial_{\nu} \tau_{\rho]}=0$ on the whole space-time $M$. This condition is known as the Frobenius condition and for a more detailed discussion of causality in the Newton-Cartan background, see Refs. $[9,12]$. A space $M$ that obeys this condition is called causal. Since $\tau_{\mu}$ is zero everywhere we can write it as $\tau_{0}=e^{-\Phi_{L}}$ where $\Phi_{L}$ is known as the Luttinger potential. In what follows we restrict ourselves to such a space-time. Since $\tau_{i}=0$ there are certain consequences for the form of the metric $h^{\mu \nu}$ thanks to the condition 


$$
\tau_{\mu} h^{\mu \nu}=0
$$

Explicitly, for $\nu=0$ this equation implies $\tau_{0} h^{00}=0$ and hence we have to have $h^{00}=0$ while for $\nu=i$ we have $\tau_{\mu} h^{\mu i}=\tau_{0} h^{0 i}=0$ which again implies that $h^{0 i}=0$. Then the action $I^{\text {sch }}$ simplifies considerably

$$
\begin{aligned}
I^{\mathrm{sch}}= & \int d^{4} x e\left(2(m-q \varphi) \rho \hat{v}^{\mu}\left(\partial_{\mu} S-q A_{\mu}\right)-2 \Phi(m-q \varphi) \rho\right. \\
& \left.-\frac{1}{4 \rho} h^{i j} \partial_{i} \rho \partial_{j} \rho-h^{i j} \rho\left(\partial_{i} S-q A_{i}\right)\left(\partial_{j} S-q A_{j}\right)\right),
\end{aligned}
$$

where $\hat{v}^{\mu}=v^{\mu}-h^{\mu \nu} M_{\nu}$ generally has all nonzero components.

Before we proceed to the Hamiltonian formulation of the theory we derive the equations of motion for $\rho$ and $S$ from Eq. (21)

$$
\begin{gathered}
\partial_{\mu}\left[e \hat{v}^{\mu}(m-q \varphi) \rho\right]-\partial_{i}\left[e \rho h^{i j}\left(\partial_{j} S-q A_{j}\right)\right]=0, \\
2 e(m-q \varphi) \hat{v}^{\mu}\left(\partial_{\mu} S-q A_{\mu}\right)-2 e \Phi(m-q \varphi) \\
+\frac{e}{4 \rho^{2}} \partial_{i} \rho \partial_{j} \rho h^{i j}+\partial_{i}\left[\frac{1}{2 \rho} e h^{i j} \partial_{j} \rho\right] \\
-e h^{i j}\left(\partial_{i} S-q A_{i}\right)\left(\partial_{j} S-q A_{j}\right)=0 .
\end{gathered}
$$

Now we are ready to proceed to the Hamiltonian formalism. From Eq. (21) we obtain the following conjugate momenta:

$$
p_{S}=\frac{\partial \mathcal{L}^{\text {sch }}}{\partial\left(\partial_{t} S\right)}=2(m-q \varphi) \rho \hat{v}^{0}, \quad p_{\rho}=\frac{\partial \mathcal{L}^{\text {sch }}}{\partial\left(\partial_{t} \rho\right)}=0 .
$$

From these two equations we see that there are two primary constraints

$\mathcal{G}_{S} \equiv p_{S}-2 e(m-q \varphi) \rho \hat{v}^{0} \approx 0, \quad \mathcal{G}_{\rho} \equiv p_{\rho} \approx 0$

while the bare Hamiltonian is equal to

$$
\begin{aligned}
H_{B}= & \int d^{d} \mathbf{x}\left(p_{\rho} \partial_{t} \rho+p_{S} \partial_{t} S-\mathcal{L}\right)=\int d^{d} \mathbf{x} \mathcal{H}_{B}, \\
\mathcal{H}_{B}= & -2 e(m-q \varphi) \rho \hat{v}^{i}\left(\partial_{i} S-q A_{i}\right)+q p_{S} A_{0} \\
& +2 e \Phi(m-q \varphi) \rho+\frac{1}{4 \rho} e h^{i j} \partial_{i} \rho \partial_{j} \rho \\
& +\rho e h^{i j}\left(\partial_{i} S-q A_{i}\right)\left(\partial_{j} S-q A_{j}\right) .
\end{aligned}
$$

We see that generally $\mathcal{G}_{S} \approx 0$ and $\mathcal{H}_{B}$ explicitly depend on time. This is not a typical situation and we discuss the theory of constraining systems with explicit time dependence in more detail in the Appendix.

As the next step we calculate the Poisson bracket between $\mathcal{G}_{S}$ and $\mathcal{G}_{\rho}$ and we obtain

$\left\{\mathcal{G}_{S}(\mathbf{x}), \mathcal{G}_{\rho}(\mathbf{y})\right\}=-2 e(m-q \varphi) \hat{v}^{0} \delta(\mathbf{x}-\mathbf{y}) \equiv \triangle_{S \rho}(\mathbf{x}, \mathbf{y})$

which shows that they are two second-class constraints. Note that the inverse matrix has the form $\triangle^{\rho S}=$ $-\frac{1}{2 e(m-q \varphi) \hat{v}^{0}} \delta(\mathbf{x}-\mathbf{y})$. As a result we can eliminate $p_{\rho}=0$ and $p_{S}=0$ from the set of canonical variables when we introduce the Dirac bracket between $\rho$ and $S$ defined as

$$
\begin{aligned}
\{\rho(\mathbf{x}), S(\mathbf{y})\}_{D}= & \{\rho(\mathbf{x}), S(\mathbf{y})\}-\int d^{d} \mathbf{z} d^{d} \mathbf{z}^{\prime}\left\{\rho(\mathbf{x}), \mathcal{G}_{S}(\mathbf{z})\right\} \triangle^{S \rho}\left(\mathbf{z}, \mathbf{z}^{\prime}\right)\left\{p_{\rho}\left(\mathbf{z}^{\prime}\right), S(\mathbf{y})\right\} \\
& -\int d^{d} \mathbf{z} d^{d} \mathbf{z}^{\prime}\left\{\rho(\mathbf{x}), p_{\rho}(\mathbf{z})\right\} \triangle^{\rho S}\left(\mathbf{z}, \mathbf{z}^{\prime}\right)\left\{\mathcal{G}_{S}\left(\mathbf{z}^{\prime}\right), S(\mathbf{y})\right\}=-\frac{1}{2 e \hat{v}^{0}(m-q \varphi)} \delta(\mathbf{x}-\mathbf{y}) .
\end{aligned}
$$

As explicitly shown in the Appendix, in the presence of the time-dependent constraints the equations of motion for the canonical variables have the form ${ }^{4}$

$$
\begin{aligned}
\partial_{t} \rho= & \left\{\rho, H_{B}\right\}_{D}-\int d^{d} \mathbf{z} d^{d} \mathbf{z}^{\prime}\left\{\rho, \mathcal{G}_{S}(\mathbf{z})\right\} \triangle^{S \rho}\left(\mathbf{z}, \mathbf{z}^{\prime}\right) \frac{\partial p_{\rho}}{\partial t}-\int d^{d} \mathbf{z} d^{d} \mathbf{z}^{\prime}\left\{\rho, p_{\rho}(\mathbf{z})\right\} \triangle^{\rho S}\left(\mathbf{z}, \mathbf{z}^{\prime}\right) \frac{\partial \mathcal{G}_{S}\left(\mathbf{z}^{\prime}\right)}{\partial t} \\
= & -\frac{1}{e \hat{v}^{0}(m-q \varphi)} \partial_{i}\left[(m-q \varphi) e \hat{v}^{i} \rho\right]-\frac{1}{e \hat{v}^{0}(m-q \varphi)} \partial_{t}\left[e(m-q \varphi) \hat{v}^{0}\right] \rho \\
& -\frac{q}{e \hat{v}^{0}(m-q \varphi)} \partial_{i}\left[e \sqrt{\rho} h^{i j} A_{j}\right]+\frac{1}{e \hat{v}^{0}(m-q \varphi)} \partial_{i}\left[e h^{i j} \rho \partial_{j} S\right]
\end{aligned}
$$

\footnotetext{
${ }^{4}$ See Eq. (A7) in the Appendix.
} 
which can be rewritten in the more symmetric form

$$
\begin{gathered}
\partial_{t}\left[e \hat{v}^{0}(m-q \varphi) \rho\right]+\partial_{i}\left[e \hat{v}^{i}(m-q \varphi) \rho\right] \\
-\partial_{i}\left[e h^{i j} \rho\left(\partial_{j} S-a A_{j}\right)\right]=0
\end{gathered}
$$

that coincides with the first equation of motion given in Eq. (22). Let us now proceed to the canonical equation of motion for $S$

$$
\begin{aligned}
\partial_{t} S= & \left\{S, H_{B}\right\}_{D}-\int d^{d} \mathbf{z} d^{d} \mathbf{z}^{\prime}\left\{S, \mathcal{G}_{S}(\mathbf{z})\right\} \triangle^{S \rho}\left(\mathbf{z}, \mathbf{z}^{\prime}\right) \frac{\partial \mathcal{G}_{\rho}}{\partial t} \\
= & -\frac{\hat{v}^{i}}{\hat{v}^{0}} \partial_{i} S+\frac{\hat{v}^{\mu}}{\hat{v}^{0}} A_{\mu}+\frac{1}{\hat{v}^{0}} \Phi-\frac{1}{8 \rho^{2} \hat{v}^{0}(m-q \varphi)} h^{i j} \partial_{i} \rho \partial_{j} \rho \\
& -\frac{1}{e \hat{v}^{0}(m-q \varphi)} \partial_{i}\left[\frac{e h^{i j}}{4 \rho} \partial_{j} \rho\right] \\
& +\frac{e}{\hat{v}^{0}(m-q \varphi)} h^{i j}\left(\partial_{i} S-q A_{i}\right)\left(\partial_{j} S-q A_{j}\right)
\end{aligned}
$$

which can be again rewritten as

$$
\begin{gathered}
2 e(m-q \varphi) \hat{v}^{\mu}\left(\partial_{\mu} S-q A_{\mu}\right)-2 e \Phi(m-q \varphi) \\
+\frac{e}{4 \rho^{2}} \partial_{i} \rho \partial_{j} \rho h^{i j}+\partial_{i}\left[\frac{1}{2 \rho} e h^{i j} \partial_{j} \rho\right] \\
-e h^{i j}\left(\partial_{i} S-q A_{i}\right)\left(\partial_{j} S-q A_{j}\right)=0
\end{gathered}
$$

which coincides with the second equation of motion given in Eq. (22).

In summary, we found the Hamiltonian formulation of the Schrödinger field in the NC background. We found that the dynamical fields are $\rho$ and $S$ which have a nonzero Dirac bracket [Eq. (27)]. Then we derived their canonical equations of motion and found that they coincide with the equations of motion derived from the Lagrangian.

\section{HAMILTONIAN FORMALISM FOR AN ELECTROMAGNETIC FIELD IN NEWTON-CARTAN GRAVITY}

In this section we focus on a canonical analysis of a nonrelativistic electromagnetic field in the NC background. We start with the simpler case of the action for nonrelativistic electrodynamics in a flat background.

\section{A. Nonrelativistic electrodynamics through null-dimensional reduction}

Following Ref. [11] we derive an action for nonrelativistic electrodynamics by performing a null reduction of the Maxwell action in one higher dimension. More precisely, let us consider the $d+2$-dimensional Maxwell action

$$
S=-\frac{1}{4} \int d t d u d^{d} \mathbf{x} F_{A B} \eta^{A C} \eta^{B D} F_{C D}
$$

where $\eta_{A B} d x^{A} d x^{B}=2 d t d u+d x^{i} d x^{i}$. Following Ref. [11] we set $A_{u}=\varphi, A_{t}=-\tilde{\varphi}, A_{i}=a_{i}$ and assume that all fields do not depend on $u$. Since the inverse metric has the form $\eta^{t u}=\eta^{u t}=1, \eta^{i j}=\delta^{i j}$ we get

$$
\begin{aligned}
F_{A B} \eta^{A C} \eta^{B D} F_{C D} & =-2\left(F_{t u}\right)^{2}+F_{i j} F^{i j}-4 F_{i u} F_{t k} \\
& =-2\left(\partial_{t} \varphi\right)^{2}-4\left(\partial_{t} a_{i}+\partial_{i} \tilde{\varphi}\right) \partial_{i} \varphi+f_{i j} F^{i j}
\end{aligned}
$$

As a result we obtain an action for nonrelativistic electrodynamics in a flat background in the form

$$
S=\int d t d^{d} \mathbf{x}\left(-\frac{1}{4} f_{i j} f^{i j}+\left(\partial_{i} \tilde{\varphi}+\partial_{t} a_{i}\right) \partial_{i} \varphi+\frac{1}{2}\left(\partial_{t} \varphi\right)^{2}\right),
$$

where $f_{i j}=\partial_{i} a_{j}-\partial_{j} a_{i}$. From the action (34) we derive the conjugate momenta ${ }^{5}$

$$
\pi^{i}=\partial_{i} \varphi, \quad p_{\tilde{\varphi}}=0, \quad p_{\varphi}=\partial_{t} \varphi
$$

so that we have the primary constraints

$$
\mathcal{G}^{i} \equiv \pi^{i}-\partial_{i} \varphi \approx 0, \quad p_{\tilde{\varphi}} \approx 0,
$$

together with the bare Hamiltonian in the form

$$
\begin{aligned}
H_{B} & =\int d^{d} \mathbf{x}\left(\pi^{i} \partial_{t} a_{i}+p_{\varphi} \partial_{t} \varphi+p_{\tilde{\varphi}} \partial_{t} \tilde{\varphi}-\mathcal{L}\right) \\
& =\int d^{d} \mathbf{x}\left(\frac{1}{4} f_{i j} f^{i j}-\partial_{i} \tilde{\varphi} \partial_{i} \varphi+\frac{1}{2} p_{\varphi}^{2}\right)
\end{aligned}
$$

and consequently the extended Hamiltonian is equal to

$$
H_{E}=H_{B}+\int d^{d} \mathbf{x}\left(\lambda_{i} \mathcal{G}^{i}+\lambda^{\tilde{\varphi}} p_{\tilde{\varphi}}\right)
$$

where $\lambda_{i}, \lambda^{\tilde{\varphi}}$ are Lagrange multipliers corresponding to the constraints $\mathcal{G}^{i} \approx 0$ and $p_{\tilde{\varphi}} \approx 0$. As the next step we have to ensure the preservation of all primary constraints. In the case of the constraint $\mathcal{G}^{i} \approx 0$ we obtain

$$
\frac{d \mathcal{G}^{i}}{d t}=\left\{\mathcal{G}^{i}, H_{E}\right\}=\partial_{k} f^{k i}-\partial_{i} p_{\varphi} \equiv \mathcal{G}_{I I}^{i} \approx 0
$$

where $\mathcal{G}_{I I}^{i} \approx 0$ are secondary constraints. In the case of the constraint $p_{\tilde{\varphi}} \approx 0$ we obtain

\footnotetext{
${ }^{5}$ In this section we do not care about upper or lower spatial indices since they are equivalent in a flat background.
} 
$\frac{d p_{\tilde{\varphi}}}{d t}=\left\{p_{\tilde{\varphi}}, H_{E}\right\}=\partial_{i} \partial^{i} \varphi=\partial_{i} \pi^{i}-\partial_{i} \mathcal{G}^{i} \approx \partial_{i} \pi^{i} \equiv \mathcal{G}_{\tilde{\varphi}}^{I I} \approx 0$

which is the generator of gauge transformations. In fact, if we define

$$
\mathbf{G}(\Lambda)=\int d^{d} \mathbf{x} \Lambda \mathcal{G}_{\tilde{\varphi}}^{I I}
$$

we obtain the standard transformation rules

$$
\left\{\mathbf{G}(\Lambda), a_{i}\right\}=\partial_{i} \Lambda, \quad\left\{\mathbf{G}(\Lambda), f_{i j}\right\}=0 .
$$

Finally we have to ensure the preservation of the constraint $\mathcal{G}_{I I}^{i} \approx 0$. To do this we have to calculate the Poisson bracket between the constraints $\mathcal{G}^{i}$ and $\mathcal{G}_{I I}^{i}$. After some calculations we obtain

$\left\{\mathcal{G}^{i}(\mathbf{x}), \mathcal{G}_{I I}^{j}(\mathbf{y})\right\}=-\partial_{k} \partial^{k} \delta(\mathbf{x}-\mathbf{y}) \delta^{i j} \equiv \triangle^{i j}(\mathbf{x}, \mathbf{y})$.

Let us introduce an inverse matrix $D_{i j}(\mathbf{x}, \mathbf{y})$ that obeys the relation

$$
\int d^{d} \mathbf{z} \triangle^{i k}(\mathbf{x}, \mathbf{z}) D_{k j}(\mathbf{z}, \mathbf{y})=\delta_{j}^{i} \delta(\mathbf{x}-\mathbf{y}) .
$$

Since $\triangle_{i j}$ is given in Eq. (43) we find that $D_{i j}$ is a solution of the equation

$$
\frac{\partial}{\partial x^{k}} \frac{\partial}{\partial x^{k}} D_{i j}(\mathbf{x}, \mathbf{y})=-\delta_{i j} \delta(\mathbf{x}-\mathbf{y}) .
$$

As the next step we determine the canonical equations of motion for $\varphi$ and $p_{\varphi}$

$$
\begin{aligned}
\partial_{t} \varphi & =\left\{\tilde{\varphi}, H_{E}\right\}=\left\{\tilde{\varphi}, H_{B}\right\}+\left\{\varphi, \int d^{d} \mathbf{z} \lambda^{i} \mathcal{G}_{i}(\mathbf{z})\right\}=p_{\varphi} \\
\partial_{t} p_{\varphi} & =\left\{p_{\varphi}, H_{E}\right\}=\left\{p_{\varphi}, H_{B}\right\}+\left\{p_{\tilde{\varphi}}, \int d^{d} \mathbf{z} \lambda^{i} \mathcal{G}_{i}(\mathbf{z})\right\} \\
& =-\partial_{k} \partial_{k} \tilde{\varphi}-\partial_{i} \lambda^{i}
\end{aligned}
$$

Finally the equation of motion for $a_{i}$ has the form

$$
\partial_{t} a_{i}=\left\{a_{i}, H_{E}\right\}=\int d^{d} \mathbf{z} \lambda_{j}(\mathbf{z})\left\{a_{i}, \mathcal{G}^{j}(\mathbf{z})\right\}=\lambda_{i}
$$

so that the equation of motion for $p_{\varphi}$ can be written as

$$
\partial_{t} p_{\varphi}=-\partial_{k}\left(\partial_{k} \tilde{\varphi}+\partial_{t} a_{k}\right)=\partial_{k} \tilde{E}_{k}
$$

where $\tilde{E}_{k}=-\partial_{t} a_{k}-\partial_{k} \tilde{\varphi}$. If we take the partial time derivative of the first equation in Eq. (46) and use the second one we obtain

$$
\partial_{t}^{2} \varphi=\partial_{t} p_{\varphi}=\partial_{k} \tilde{E}_{k}
$$

in agreement with Eq. (2.11) in Ref. [11]. Further, if we apply the partial derivative $\partial_{i}$ to the first equation in Eq. (46) we obtain

$$
\partial_{i} \partial_{t} \varphi=\partial_{i} p_{\varphi}=\partial_{k} f^{k i}
$$

which agrees with the second equation in Eq. (2.3) in Ref. [11]. In the same way we find that the divergence of $\mathcal{G}^{i}=0$ implies

$$
\partial_{i} \mathcal{G}^{i}=\mathcal{G}_{\tilde{\varphi}}^{I I}-\partial_{i} \partial^{i} \varphi \approx-\partial_{i} \partial^{i} \tilde{\varphi}=0
$$

which agrees with the first equation in Eq. (2.3) in Ref. [11]. Finally we should determine the Lagrange multiplier $\lambda_{i}$ using the requirement of the preservation of the constraint $\mathcal{G}_{I I}^{i}$ but this is not necessary since we know that $\lambda_{i}=\partial_{t} a_{i}$. On the other hand since $\mathcal{G}^{i} \approx 0$ and $\mathcal{G}_{I I}^{i} \approx 0$ are two second-class constraints they can be explicitly solved for $\pi^{i}$ and $a_{i}$. In other words the only dynamical variables are $\varphi$ and its conjugate momentum $p_{\varphi}$.

\section{B. Null reduction of Maxwellian electromagnetism in the $\mathrm{NC}$ background}

We determine the action for an electromagnetic field in the Newton-Cartan background again with the help of nulldimensional reduction, following Ref. [11]. We start with the action for an electromagnetic field in $d+2$ dimensions which has the form

$$
S=-\frac{1}{4} \int d^{d+2} x \sqrt{-\gamma} F_{A B} \gamma^{A C} \gamma^{B D} F_{C D}
$$

Our goal is to dimensionally reduce this action along a null isometry so we assume that $A_{M}$ do not depend on $u$. We further write $A_{M}=\left(A_{u}, A_{\mu}\right)$ and define $A_{\mu} \equiv \varphi$. Since the gauge field transforms under $U(1)$ transformations as

$$
A_{A}^{\prime}=A_{A}+\partial_{A} \Lambda
$$

it is clear that $\varphi$ is invariant under gauge transformations since $\Lambda$ does not depend on $u$. On the other hand the gauge field $A_{\mu}$ transforms as

$$
A_{\mu}^{\prime}=A_{\mu}+\partial_{\mu} \Lambda
$$

In order to perform null-dimensional reductions we use the components of the inverse metric given in Eq. (13) and we obtain the action in the form 


$$
\begin{aligned}
S= & \int d^{d+1} x e\left(-\frac{1}{4} F_{\mu \nu} h^{\mu \rho} h^{\nu \sigma} F_{\rho \sigma}-\Phi \partial_{\mu} \varphi h^{\mu \nu} \partial_{\nu} \varphi\right. \\
& \left.+\frac{1}{2}\left(\hat{v}^{\mu} \partial_{\mu} \varphi\right)^{2}-\hat{v}^{\nu} F_{\nu \mu} h^{\mu \sigma} \partial_{\sigma} \varphi\right),
\end{aligned}
$$

where

$$
F_{\mu \nu}=\partial_{\mu} A_{\nu}-\partial_{\nu} A_{\mu} .
$$

It is convenient to use a slightly different form for the action which depends on $v^{\mu}$ instead of $\hat{v}^{\mu}$. Following Ref. [11] we introduce the vector field $\bar{A}_{\mu}$ defined as

$$
A_{\mu}=\bar{A}_{\mu}-\varphi M_{\mu}
$$

Performing this substitution in the action (55) we find

$$
\begin{aligned}
S= & \int d^{d+1} x e\left(-\frac{1}{4} h^{\mu \rho} h^{\nu \sigma} \bar{F}_{\mu \nu} \bar{F}_{\rho \sigma}-h^{\mu \nu} v^{\rho} \bar{F}_{\rho \nu} \partial_{\mu} \varphi\right. \\
& \left.+\frac{1}{2}\left(v^{\mu} \partial_{\mu} \varphi\right)^{2}\right),
\end{aligned}
$$

where

$$
\bar{F}_{\mu \nu}=\partial_{\mu} \bar{A}_{\nu}-\partial_{\nu} \bar{A}_{\nu}-\varphi\left(\partial_{\mu} M_{\nu}-\partial_{\nu} M_{\mu}\right) .
$$

The action (58) will be the starting point for the Hamiltonian formulation of the theory. As we argued above we restrict ourselves to a causal space-time with nonzero $\tau_{0}$ only. Then $v^{\mu}$ generally has all nonzero components with $v^{0}=-\tau_{0}$. In the case of a causal space-time the action has the form

$$
\begin{aligned}
S= & \int d^{d+1} x e\left(-\frac{1}{4} \bar{F}_{i j} h^{i k} h^{j l} \bar{F}_{k l}-\Phi \partial_{i} \varphi h^{i j} \partial_{j} \varphi\right. \\
& \left.+\frac{1}{2}\left(v^{\mu} \partial_{\mu} \varphi\right)^{2}-v^{\nu} \bar{F}_{\nu i} h^{i j} \partial_{j} \varphi\right) .
\end{aligned}
$$

Note that from this action we also obtain the equations of motion in the form

$$
\begin{aligned}
& 2 \partial_{i}\left[e \Phi h^{i j} \partial_{j} \varphi\right]-\partial_{\mu}\left[e v^{\mu} v^{\nu} \partial_{\nu} \varphi\right]+\partial_{j}\left[e v^{\nu} \bar{F}_{\nu i} h^{i j}\right] \\
& \quad+\frac{1}{2} e\left(\partial_{i} M_{j}-\partial_{j} M_{i}\right) h^{i k} h^{j l} \bar{F}_{k l} \\
& \quad+e v^{0}\left(\partial_{0} M_{i}-\partial_{i} M_{0}\right) h^{i j} \partial_{j} \varphi \\
& \quad+e v^{k}\left(\partial_{k} M_{i}-\partial_{i} M_{k}\right) h^{i j} \partial_{j} \varphi=0, \\
& \partial_{j}\left[e h^{i l} \bar{F}_{l k} h^{k j}\right]+\partial_{0}\left[e v^{0} h^{i j} \partial_{j} \varphi\right]+\partial_{k}\left[e v^{k} h^{i j} \partial_{j} \varphi\right] \\
& \quad-\partial_{k}\left[e v^{i} h^{k j} \partial_{j} \varphi\right]=0, \\
& \partial_{i}\left[e v^{0} h^{i j} \partial_{j} \varphi\right]=0 .
\end{aligned}
$$

Let us now proceed to the canonical analysis. From Eq. (60) we obtain the conjugate momenta

$$
\begin{aligned}
\pi^{i} & =\frac{\partial \mathcal{L}}{\partial\left(\partial_{t} \bar{A}_{i}\right)}=-e v^{0} h^{i j} \partial_{j} \varphi, \quad \pi^{0}=\frac{\partial \mathcal{L}}{\partial\left(\partial_{t} \bar{A}_{0}\right)} \approx 0 \\
p_{\varphi} & =\frac{\partial \mathcal{L}}{\partial\left(\partial_{t} \varphi\right)}=e v^{0}\left(v^{\mu} \partial_{\mu} \varphi\right)
\end{aligned}
$$

so that we have the following explicitly time-dependent primary constraints:

$$
\mathcal{G}^{i} \equiv \pi^{i}+e v^{0} h^{i j} \partial_{j} \varphi \approx 0
$$

together with the familiar constraint $\mathcal{G}^{0} \equiv \pi^{0} \approx 0$. Further, with the help of Eq. (62), we obtain the bare Hamiltonian in the form

$$
\begin{aligned}
H_{B}= & \int d^{d} \mathbf{x}\left(e \frac{1}{4} \bar{F}_{i j} h^{i k} h^{j l} \bar{F}_{k l}+e \Phi \partial_{i} \varphi h^{i j} \partial_{j} \varphi\right. \\
& +e v^{k} \bar{F}_{k i} h^{i j} \partial_{j} \varphi+\frac{1}{2 e}\left(\tau_{0}\right)^{2} p_{\varphi}^{2}+\tau_{0} v^{i} \partial_{i} \varphi p_{\varphi} \\
& \left.-A_{0} \partial_{i} \pi^{i}+\pi^{i} \varphi\left(\partial_{0} M_{i}-\partial_{i} M_{0}\right)\right) .
\end{aligned}
$$

Now we have to analyze the requirement of the preservation of the primary constraints $\mathcal{G}^{i} \approx 0, \mathcal{G}^{0} \approx 0$. Note that the extended Hamiltonian has the form

$$
H_{E}=H_{B}+\int d^{d} \mathbf{x}\left(\lambda_{i} \mathcal{G}^{i}+\lambda_{0} \pi^{0}\right)
$$

In the case of $\mathcal{G}^{0} \approx 0$ we obtain that the requirement of its preservation during the time development of the system implies the standard Gauss' law constraint

$$
\mathcal{G}^{I I} \equiv \partial_{i} \pi^{i} \approx 0,
$$

while in the case of $\mathcal{G}^{i}$ we get

$$
\begin{aligned}
\frac{d \mathcal{G}^{i}}{d t}= & \frac{\partial \mathcal{G}^{i}}{\partial t}+\left\{\mathcal{G}^{i}, H_{E}\right\} \\
= & \partial_{t}\left(e v^{0} h^{i j}\right) \partial_{j} \varphi-\partial_{k}\left[e h^{i k} \bar{F}_{k l} h^{l j}\right]+\partial_{k}\left(e v^{k} h^{\mathrm{in}} \partial_{n} \varphi\right) \\
& -\partial_{m}\left(e v^{i} h^{m n} \partial_{n} \varphi\right)+e v^{0} h^{i j} \partial_{j}\left[\frac{1}{e}\left(\tau_{0}\right)^{2} p_{\varphi}\right] \\
& +e v^{0} h^{i j} \partial_{j}\left[\tau_{0} v^{m} \partial_{m} \varphi\right] \equiv \mathcal{G}_{I I}^{i} \approx 0
\end{aligned}
$$

where we used the fact that $\left\{\mathcal{G}^{i}(\mathbf{x}), \mathcal{G}^{j}(\mathbf{y})\right\}=0$. We see that the requirement of the preservation of the constraints $\mathcal{G}^{i} \approx 0$ implies that the second set of constraints $\mathcal{G}_{I I}^{i} \approx 0$. It is again easy to see that $\mathcal{G}^{i} \approx 0, \mathcal{G}_{I I}^{j} \approx 0$ are two sets of second-class constraints with a rather complicated Poisson bracket between them. Then it is difficult to determine Lagrange multipliers $\lambda_{i}$ from the requirement of the preservation of the constraints $\mathcal{G}_{I I}^{i} \approx 0$ during the time evolution of the system. On the other hand, as we will show below, these 
Lagrange multipliers can be determined with the help of the equations of motion for $\bar{A}_{i}$. Further, it is easy to see that $\mathcal{G}^{0}$ and $\mathcal{G}^{I I} \approx 0$ are first-class constraints where $\mathcal{G}^{I I} \approx 0$ is the generator of gauge transformations.

As we argued above $\mathcal{G}^{i} \approx 0, \mathcal{G}_{I I}^{i} \approx 0$ are two sets of second-class constraints where $\mathcal{G}^{i}=0$ can be solved for $\pi^{i}$ while $\mathcal{G}_{I I}^{i}=0$ can be solved for $\bar{A}_{i}$ at least in principle. On the other hand when we try to write equations of motion for $\varphi$ and $p_{\varphi}$ it is convenient to express the Lagrange multipliers $\lambda_{i}$ as functions of the nondynamical variable $\bar{A}_{i}$ using its equation of motion

$$
\partial_{t} \bar{A}_{i}=\left\{\bar{A}_{i}, H_{E}\right\}=\partial_{i} A_{0}+\lambda_{i}+\varphi\left(\partial_{0} M_{i}-\partial_{i} M_{0}\right)
$$

which implies that $\lambda_{i}=\bar{F}_{0 i}$. Then we can write the canonical equations of motion for $\varphi$ and $p_{\varphi}$ as

$$
\begin{aligned}
\partial_{t} \varphi= & \left\{\varphi, H_{B}\right\}+\int d^{d} \mathbf{z} \lambda_{i}\left\{\varphi, \mathcal{G}^{i}(\mathbf{z})\right\}=\frac{1}{e\left(v^{0}\right)^{2}} p_{\varphi}+\tau_{0} v^{i} \partial_{i} \varphi \\
\partial_{t} p_{\varphi}= & \left\{p_{\varphi}, H_{B}\right\}+\int d^{d} \mathbf{z} \lambda_{i}(\mathbf{z})\left\{p_{\varphi}, \mathcal{G}^{i}(\mathbf{z})\right\} \\
= & \frac{1}{2} e\left(\partial_{i} M_{j}-\partial_{j} M_{i}\right) h^{i k} h^{j l} \bar{F}_{k l}-\pi^{i}\left(\partial_{0} M_{i}-\partial_{i} M_{0}\right)+\partial_{m}\left(\lambda_{j} h^{j m} e\right) \\
& +2 \partial_{i}\left[e \Phi h^{i j} \partial_{j} \varphi\right]+\partial_{j}\left[e v^{k} \bar{F}_{k i} h^{i j}\right]+\partial_{i}\left[\tau_{0} v^{i} p_{\varphi}\right]+e v^{k}\left(\partial_{k} M_{i}-\partial_{i} M_{k}\right) h^{i j} \partial_{j} \varphi \\
\approx & \frac{1}{2} e\left(\partial_{i} M_{j}-\partial_{j} M_{i}\right) h^{i k} h^{j l} \bar{F}_{k l}+v^{0} e h^{i j} \partial_{j} \varphi\left(\partial_{0} M_{i}-\partial_{i} M_{0}\right)+\partial_{m}\left(\bar{F}_{0 j} h^{j m} e v^{0}\right) \\
& +2 \partial_{i}\left[e \Phi h^{i j} \partial_{j} \varphi\right]+\partial_{j}\left[e v^{k} \bar{F}_{k i} h^{i j}\right]+\partial_{i}\left[\tau_{0} v^{i} p_{\varphi}\right]+e v^{k}\left(\partial_{k} M_{i}-\partial_{i} M_{k}\right) h^{i j} \partial_{j} \varphi .
\end{aligned}
$$

If we combine these two equations together we obtain

$$
\begin{aligned}
\partial_{t}\left(e\left(v^{0}\right)^{2} \partial_{t} \varphi\right)= & \partial_{t} p_{\varphi}-\partial_{t}\left(e v^{0} v^{i} \partial_{i} \varphi\right) \\
= & \frac{1}{2} e\left(\partial_{i} M_{j}-\partial_{j} M_{i}\right) h^{i k} h^{j l} \bar{F}_{k l}+e v^{0} h^{i j} \partial_{j} \varphi\left(\partial_{0} M_{i}-\partial_{i} M_{0}\right)+\partial_{m}\left(\bar{F}_{0 j} h^{j m} e v^{0}\right) \\
& +2 \partial_{i}\left[e \Phi h^{i j} \partial_{j} \varphi\right]+\partial_{j}\left[e v^{k} \bar{F}_{k i} h^{i j}\right]-\partial_{i}\left[e v^{i} v^{0} \partial_{t} \varphi\right]+e v^{k}\left(\partial_{k} M_{i}-\partial_{i} M_{k}\right) h^{i j} \partial_{j} \varphi
\end{aligned}
$$

which coincides with the first equation in Eq. (61). Further, it is easy to see that the second equation in Eq. (61) coincides with the secondary constraint $\mathcal{G}_{I I}^{i}=0$. Finally, the last equation in Eq. (61) is equivalent to the combination of the primary constraints $\mathcal{G}^{I I}$ and $\mathcal{G}^{i}$ since

$$
\mathcal{G}^{I I}=\partial_{i}\left[\mathcal{G}^{i}-e v^{0} h^{i j} \partial_{j} \varphi\right]=-\partial_{i}\left[e v^{0} h^{i j} \partial_{j} \varphi\right]=0 .
$$

In summary, we have shown that the canonical equations of motion and constraints reproduce the Lagrangian equations of motion. We have also determined the physical d.o.f. of nonrelativistic electrodynamics and we have shown that there are only two phase space physical d.o.f. left corresponding to $\varphi$ and $p_{\varphi}$.

\section{ACKNOWLEDGMENTS}

I would like to thank M. Chaichian for his comments and remarks. This work was supported by the Grant Agency of the Czech Republic under the Grant No. P201/12/G028.

\section{APPENDIX: SYSTEMS WITH EXPLICIT TIME-DEPENDENT CONSTRAINTS}

Let us consider a phase space system with variables $p_{m}$, $q^{m}, m=1, \ldots, N$, a bare Hamiltonian $H_{B}$ and a set of primary constraints $\phi_{j}=\phi_{j}(p, q, t), j=1, \ldots, J$, that explicitly depend on time $t$. Then the phase space action with the primary constraints included has the form

$$
S=\int d t\left(p_{m} \dot{q}^{m}-H_{B}-\lambda^{j} \phi_{j}\right)
$$

where $\lambda^{j}$ are independent variables known as Lagrange multipliers. By varying the action with respect to $p_{m}, q^{m}$ and $\lambda^{j}$ we obtain the following set of equations of motion:

$$
\begin{aligned}
\dot{q}^{m}-\frac{\partial H_{B}}{\partial p_{m}}-\lambda^{j} \frac{\partial \phi_{j}}{\partial p_{m}} & =0, \\
-\dot{p}_{m}-\frac{\partial H_{B}}{\partial q^{m}}-\lambda^{j} \frac{\partial \phi_{j}}{\partial q^{m}} & =0, \\
\phi_{j} & \approx 0 .
\end{aligned}
$$

By introducing the standard Poisson bracket they can be written as

$$
\begin{aligned}
& \dot{q}^{m}=\left\{q^{m}, H_{B}\right\}+\lambda^{j}\left\{q^{m}, \phi_{j}\right\}, \\
& \dot{p}_{m}=\left\{p_{m}, H_{B}\right\}+\lambda^{j}\left\{p_{m}, \phi_{j}\right\}, \quad \phi_{j} \approx 0 .
\end{aligned}
$$


In order to determine the Lagrange multipliers $\lambda^{j}$ we demand that the constraints $\phi_{j}=0$ are preserved during the time evolution of the system. We note that it is clear from the form of the equations of motion written above that we have to first calculate the Poisson bracket between the canonical variables and $\phi_{j}$ and then we can impose the condition $\phi_{j}=0$. This is the reason why we write $\phi_{j} \approx 0$ instead of $\phi_{j}=0$. Now the time evolution of the constraint $\phi_{i}$ is equal to

$$
\begin{aligned}
\dot{\phi}_{i} & =\frac{\partial \phi_{i}}{\partial t}+\frac{\partial \phi_{i}}{\partial q^{m}} q^{i m}+\frac{\partial \phi_{i}}{\partial p_{m}} \dot{p}_{m} \\
& =\frac{\partial \phi_{i}}{\partial t}+\left\{\phi_{i}, H_{B}\right\}+\left\{\phi_{i}, \phi_{j}\right\} \lambda^{j} .
\end{aligned}
$$

If we impose the condition that the constraint $\phi_{i}$ is preserved during the time evolution of the system we find that the conditions $\dot{\phi}_{i}=0$ provide $J$ equations for $J$ unknown $\lambda^{i}$. Let us now presume the nondegenerate case when $\left\{\phi_{i}, \phi_{j}\right\}=\triangle_{i j}$ is a nonsingular matrix so that it has an inverse $\triangle^{j k}, \triangle_{i j} \triangle^{j k}=\delta_{i}^{k}$. Then Eq. (A4) can be solved as

$$
\lambda^{i}=-\triangle^{i k}\left(\frac{\partial \phi_{k}}{\partial t}+\left\{\phi_{k}, H_{B}\right\}\right)
$$

As a result we find that the time evolution of the phase space variables $q^{m}$ and $p_{m}$ is governed by the equations

$$
\begin{aligned}
& \dot{q}^{m}=\left\{q^{m}, H_{B}\right\}-\left\{q^{m}, \phi_{i}\right\} \triangle^{i j}\left\{\phi_{j}, H_{B}\right\}-\left\{q^{m}, \phi_{i}\right\} \triangle^{i j} \frac{\partial \phi_{j}}{\partial t}, \\
& \dot{p}_{m}=\left\{p_{m}, H_{B}\right\}-\left\{p_{m}, \phi_{i}\right\} \triangle^{i j}\left\{\phi_{j}, H_{B}\right\}-\left\{p_{m}, \phi_{i}\right\} \triangle^{i j} \frac{\partial \phi_{j}}{\partial t}, \\
& \phi_{j} \approx 0
\end{aligned}
$$

which can be written in the equivalent form

$$
\begin{aligned}
\dot{q}^{m} & =\left\{q^{m}, H_{B}\right\}_{D}-\left\{q^{m}, \phi_{i}\right\} \triangle^{i j} \frac{\partial \phi_{j}}{\partial t}, \\
\dot{p}_{m} & =\left\{p_{m}, H_{B}\right\}_{D}-\left\{p_{m}, \phi_{i}\right\} \triangle^{i j} \frac{\partial \phi_{j}}{\partial t}, \\
\phi_{j} & \approx 0,
\end{aligned}
$$

where we introduced the Dirac bracket between two phase space functions defined as $\{X, Y\}_{D}=\{X, Y\}-$ $\left\{X, \phi_{i}\right\} \triangle^{i j}\left\{\phi_{j}, Y\right\}$.

\section{Secondary time-dependent constraints}

Let us now consider the situation where the primary constraints $\phi_{j}(p, q, t)$ have weakly vanishing Poisson brackets among themselves. Then the requirement that they are preserved during the time development of the system has the form

$$
\begin{aligned}
\frac{d \phi_{i}}{d t}= & \frac{\partial \phi_{i}}{\partial t}+\left\{\phi_{i}, H_{B}\right\}+\lambda^{j}\left\{\phi_{i}, \phi_{j}\right\} \approx \frac{\partial \phi_{i}}{\partial t} \\
& +\left\{\phi_{i}, H_{B}\right\} \equiv \phi_{i}^{I I}(p, q, t) \approx 0,
\end{aligned}
$$

where now $\phi_{i}^{I I}(p, q, t)$ are secondary constraints. Finally we have to ensure the preservation of the secondary constraints which implies

$$
\frac{d \phi_{i}^{I I}}{d t}=\frac{\partial \phi_{i}^{I I}}{\partial t}+\left\{\phi_{i}^{I I}, H_{B}\right\}+\lambda^{j}\left\{\phi_{i}^{I I}, \phi_{j}\right\} \approx 0 .
$$

We will assume that the matrix $\left\{\phi_{i}^{I I}, \phi_{j}\right\} \equiv \triangle_{i j}$ is nonsingular and hence the equation above can be solved for $\lambda^{i}$ as

$$
\lambda^{i}=-\triangle^{i j}\left(\frac{\partial \phi_{j}^{I I}}{\partial t}+\left\{\phi_{j}^{I I}, H_{B}\right\}\right)
$$

and hence the equation of motion for $p^{m}, q_{m}$ has the form

$$
\begin{aligned}
\frac{d q^{m}}{d t}= & \left\{q^{m}, H_{B}\right\}+\lambda^{j}\left\{q^{m}, \lambda_{j}\right\}=\left\{q^{m}, H_{B}\right\} \\
& -\left\{q^{m}, \phi_{i}\right\} \triangle^{i j}\left(\frac{\partial \phi_{j}^{I I}}{\partial t}+\left\{\phi_{j}^{I I}, H_{B}\right\}\right)
\end{aligned}
$$

and an equivalent one for $p_{m}$.

Let us outline the results of the analysis performed in this Appendix. We have shown that in the case of explicit time-dependent constraints, either primary or secondary, there are additional terms in the equations of motion for canonical variables which are proportional to the explicit time derivative of these constraints. These terms are crucial for the equivalence between the Lagrangian and Hamiltonian equations of motion. 
[1] D. T. Son, Newton-Cartan geometry and the quantum Hall effect, arXiv:1306.0638.

[2] M. H. Christensen, J. Hartong, N. A. Obers, and B. Rollier, Torsional Newton-Cartan geometry and Lifshitz holography, Phys. Rev. D 89, 061901 (2014).

[3] M.H. Christensen, J. Hartong, N. A. Obers, and B. Rollier, Boundary stress-energy tensor and Newton-Cartan geometry in Lifshitz holography, J. High Energy Phys. 01 (2014) 057.

[4] M. Geracie, D. T. Son, C. Wu, and S. F. Wu, Spacetime symmetries of the quantum Hall effect, Phys. Rev. D 91, 045030 (2015).

[5] K. Jensen, On the coupling of Galilean-invariant field theories to curved spacetime, SciPost Phys. 5, 011 (2018).

[6] J. Hartong, E. Kiritsis, and N. A. Obers, Schrödinger invariance from Lifshitz isometries in holography and field theory, Phys. Rev. D 92, 066003 (2015).

[7] J. Hartong, E. Kiritsis, and N. A. Obers, Field theory on Newton-Cartan backgrounds and symmetries of the Lifshitz vacuum, J. High Energy Phys. 08 (2015) 006.

[8] M. Taylor, Lifshitz holography, Classical Quantum Gravity 33, 033001 (2016).

[9] M. Geracie, K. Prabhu, and M. M. Roberts, Curved nonrelativistic spacetimes, Newtonian gravitation and massive matter, J. Math. Phys. 56, 103505 (2015).
[10] E. Bergshoeff, J. Rosseel, and T. Zojer, Non-relativistic fields from arbitrary contracting backgrounds, Classical Quantum Gravity 33, 175010 (2016).

[11] G. Festuccia, D. Hansen, J. Hartong, and N. A. Obers, Symmetries and couplings of non-relativistic electrodynamics, J. High Energy Phys. 11 (2016) 037.

[12] M. Geracie, K. Prabhu, and M. M. Roberts, Fields and fluids on curved non-relativistic spacetimes, J. High Energy Phys. 08 (2015) 042.

[13] G. Festuccia, D. Hansen, J. Hartong, and N. A. Obers, Torsional Newton-Cartan geometry from the Noether procedure, Phys. Rev. D 94, 105023 (2016).

[14] R. Basu and U. N. Chowdhury, Dynamical structure of Carrollian electrodynamics, J. High Energy Phys. 04 (2018) 111.

[15] C. Duval, G. Burdet, H. P. Kunzle, and M. Perrin, Bargmann structures and Newton-Cartan theory, Phys. Rev. D 31, 1841 (1985).

[16] B. Julia and H. Nicolai, Null Killing vector dimensional reduction and Galilean geometrodynamics, Nucl. Phys. B439, 291 (1995).

[17] D. M. Gitman and I. V. Tyutin, Quantization of Fields with Constraints (Springer, New York, 1990). 